\title{
Bacteria find work as amateur chemists
}

New evidence demonstrating that certain bacterial strains can synthesize semiconductor nanocrystals through endogenous pathways may open the door to new strategies for the biosynthesis of unnatural materials and compounds.

Semiconductor nanocrystals are finding use in a wide variety of scientific applications, although biologists are probably most familiar with their 'quantum dot' incarnation. Currently, these are produced by chemical syntheses in specialized laboratories. However, in recent years, University of Texas investigators Brent Iverson and Angela Belcher (now at MIT) have become interested in the possibility of using biological systems to assemble these specialized reagents.

Biosynthesis of such crystals is not an entirely new concept, as earlier studies have demonstrated nanocrystal biosynthesis in yeast (Dameron et al., 1989). Additionally, an earlier collaboration between Belcher and Iverson identified peptides that, when expressed on the surface of M13 bacteriophage, were capable of nucleating the formation of semiconductor nanowires from zinc or cadmium sulfide (Mao et al., 2003). Iverson, Belcher and colleagues now sought to expand this strategy to bacteria and designed a system for expressing bacterial surface peptides that they hoped would mirror the effects observed in viruses. What they got instead, according to Iverson, was a surprise: "The negative controls worked too..., and what we realized was that Escherichia coli have this ability - and it had nothing to do with the peptide that we were putting on the surface of the bacteria."

Indeed, hours after incubating bacteria of the ABLE C strain with cadmium chloride and sodium sulfide, the investigators observed densely packed deposits of nanocrystals by electron microscopy (Sweeney et al., 2004). Further analysis revealed that this process takes place primarily in stationary-phase cells (Fig. 1) and that only certain bacterial strains are capable of efficient nanocrystal formation.

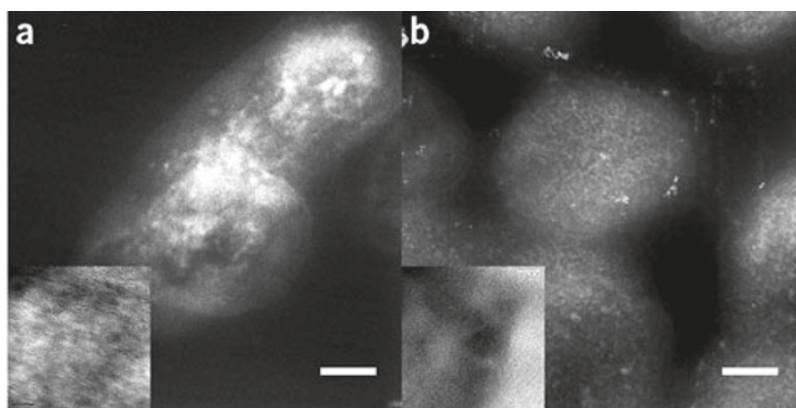

Figure 1 | Electron microscope images show that ABLE C cells in stationary phase (a) contain substantial deposits of cadmium sulfide nanocrystals, whereas cells in late log phase (b) do not. Bar indicates $200 \mathrm{~nm}$; inset depicts enlarged image of cell. Reprinted from Chemistry $\&$ Biology with permission from Elsevier.

\section{SPECTROSCOPY}

\section{METABOLITE DISCOVERY GOES GLOBAL}

With a new strategy for global metabolite profiling that identifies all substrates for a given enzyme comes a link between proteome and metabolome.

Let's imagine the following scenario: a research group starts working on a particular enzyme with the question, "what are the substrates my enzyme is acting on?" As fundamental as this question is, no method for obtaining a comprehensive answer has yet been described. Alan Saghatelian and his colleagues in Benjamin Cravatt's lab at the Scripps Research Institute sought to change that. They developed a strategy that uses untargeted liquid chromatography-mass spectrometry (LC-MS) to analyze all metabolites generated by a particular enzyme. Their approach, which they call discovery metabolite profiling (DMP), is described in a recent paper in Biochemistry (Saghatelian et al., 2004).

DMP is based on the comparison of changes in metabolite levels in the presence or the absence of an enzyme. The lipase fatty acid amide hydrolase (FAAH) was the enzyme of choice for Cravatt's group. They extracted lipophilic compounds from brains and spinal cords of wild-type or FAAH knockout mice and compared the LC-MS spectra over a wide mass range. Peaks seen
Beyond these determinations, however, this biosynthetic process remains poorly understood, and initial experiments investigating links between cellular thiol content and crystal formation proved inconclusive. "All possibilities are open for discussion," says Iverson, although early indications suggest that some endogenous molecule is initiating a cytosolic nucleation process. "We're trying to isolate that critical piece in the knockout but not in the wild-type sample represented FAAH substrates.

The originality and strength of DMP lies in the fact that it is untargeted and uses no standard for quantification of compounds, thus allowing a comprehensive analysis of the whole metabolome. In targeted LC-MS methods, researchers start with a known metabolite of interest and use a standard that is similar in mass. This allows absolute quantitative measurements, but eliminates the possibility of detecting new substrates.

Saghatelian was first able to identify known substrates with DMP, thus validating the method. What came as a surprise was that he also discovered a new class of metabolites, taurineconjugated fatty acids, which have not been associated with FAAH previously. This finding is intriguing for several reasons. First, it demonstrates the ability of DMP to identify a set of completely unexpected substrates. Second, it underscores the importance of a method that starts with an in vivo sample to characterize the enzymatic regulation of metabolites. The taurine conjugates proved to be extremely poor substrates for FAAH in vitro and their connection to FAAH would never have been 
that's missing—what's the molecule that's causing it—because we assume that it's nucleation."

Emory University professor Shuming Nie, who has worked extensively with quantum dots, lauds the potential of this system: "It's a really innovative, excellent approach to use biological systems to synthesize these semiconductor nanocrystals, [and] it shows that these nanocrystals are intrinsically compatible with biological systems." Nonetheless, he warns that the quality of such biosynthetic preparations at present "can not really compete with the quality — either in size, distribution or optical efficiency — of materials synthesized in the laboratory." The authors agree that this is only the beginning, and Belcher says that optimizing the quality and size distribution of these biosynthetic nanocrystals are top priorities for future investigation.

Nevertheless, these findings provide encouraging evidence of the potential benefits that could be reaped using bacteria for the synthesis of a variety of products and materials. "I think that it's an exciting new process, just in terms of harnessing the potential of organisms to make materials that they normally don't make," says Belcher. Iverson concurs and sees broad possibilities for optimizing such biological pathways: "This [process] is clearly happening without intervention, and I think there is absolutely no reason to believe that you can't start intervening by putting known nucleators in and controlling their expression and abundance, and try to do things in a very rational way... because these are really good little reactors."

\section{Michael Eisenstein}

\section{RESEARCH PAPERS}

Sweeney, R.Y. et al. Bacterial biosynthesis of cadmium sulfide nanocrystals. Chem. Biol. 11, 1553-1559 (2004).

Mao, C. et al. Viral assembly of oriented quantum dot nanowires. Proc. Natl. Acad. Sci. USA 100, 6946-6951 (2003).

Dameron, C.T. et al. Biosynthesis of cadmium sulphide quantum semiconductor crystallites. Nature 338, 596-597 (1989).

suspected based on in vitro data.

To show the potential of DMP even more clearly, Saghatelian plans to expand it in several ways. As the method requires an enzyme-depleted sample, he wants to use RNA interference or chemical inhibitors, rather than the more cumbersome gene knockout method, to reduce enzyme levels. To show that DMP is reproducible and not restricted to discovering FAAH metabolites, he intends to test it with other lipases. Finally, by modifying the way samples are fractionated and prepared for LC-MS, Saghatelian will no longer be restricted to lipophilic compounds but will also be able to include water-soluble metabolites in the analysis, thereby expanding the list of enzymes DMP can be used for.

With all its potential, this method will most likely not be confined to one laboratory for long, and Cravatt has high hopes for the future of DMP: "We anticipate that these findings will inspire every scientist to apply DMP to his or her enzyme of interest to elucidate its endogenous function and facilitate its integration into larger metabolic networks in the cell."

\section{Nicole Rusk}

\section{RESEARCH PAPERS}

Saghatelian, A. et al. Assignment of endogenous substrates to enzymes by global metabolite profiling. Biochemistry 43, 14332-14339 (2004).

\section{NEWS IN BRIEF}

\section{CELL BIOLOGY}

\section{Light-activated ion channels for remote control of neuronal firing}

Banghart et al. describe a specialized chemical gating molecule capable of undergoing light-induced photoisomerization, resulting in the reversible inhibition of ion channel function. They successfully demonstrate the use of the compound, MAL-AZO-QA, to block the action of the voltage- gated Shaker potassium channel in Xenopus laevis oocytes and cultured rat hippocampal neurons. Banghart, M. et al. Nat. Neurosci., published online 21 November 2004.

\section{MICROARRAYS}

\section{Printed covalent glycan array for ligand profiling of diverse glycan binding proteins}

Several different kinds of glycan-based arrays have been developed to assess the specificities of glycan binding proteins (GBPs). Blixt et al. present an approach that enables the generation of such arrays by the same robotic printing process used for DNA arrays. Studies conducted with such arrays show high sensitivity and reproducibility, with consistently low background.

Blixt, O. et al. Proc. Natl. Acad. Sci. USA 101, 17033-17038 (2004).

\section{GENOMICS}

\section{DNA methylation profiling of the human major histocompatibility complex: A pilot study for the human epigenome project}

The Human Epigenome Project was launched just over a year ago with the aim of assembling an atlas of DNA methylation within the regulatory regions of all human genes. Rakyan et al. present the outcome of the pilot phase of this project, a DNA methylation profile for the human major histocompatibility complex. Rakyan, V.K. et al. PLoS Biology, published online 23 November 2004.

\section{Baculovirus expression system for heterologous multiprotein complexes}

Baculoviral vectors can contain large amounts of exogenous DNA, making them appealing candidates for the assembly of multicistronic expression constructs. Berger et al. describe a 'multiplication module' strategy for the rapid, iterative assembly of multiple promoter-gene sets within a transfer vector, which can then be recombined into a bacmid for efficient expression. Berger, I. et al. Nat. Biotechnol., published online 28 November 2004.

\section{RNA INTERFERENCE}

\section{Highly efficient small interfering RNA delivery to primary mammalian neurons induces microRNA-like effects before mRNA degradation}

Although RNA interference (RNAi) can be an extremely powerful tool for gene silencing, its use in nervous system studies has been limited by the difficulties involved in transfecting neurons. By conjugating the vector peptide Penetratin 1 to their small interfering RNAs, Davidson et al. achieve effective and nontoxic delivery into cultured neurons, and demonstrate successful RNAi for several genes.

Davidson, T.J. et al. J. Neurosci. 24, 10040-10046 (2004). 